\title{
Feasibility of motivational interviewing delivered by a glaucoma educator to improve medication adherence
}

This article was published in the following Dove Press journal:

Clinical Ophthalmology

22 September 2010

Number of times this article has been viewed

\author{
Paul F Cook' \\ Robert W Bremer ${ }^{2}$ \\ AJ Ayala ${ }^{4}$ \\ Malik Y Kahook ${ }^{3}$ \\ 'College of Nursing, ${ }^{2}$ Department \\ of Psychiatry, ${ }^{3}$ Department of \\ Ophthalmology, School of Medicine, \\ University of Colorado Denver, \\ Aurora, CO, USA; ${ }^{4}$ Rocky Mountain \\ Lions Eye Institute, University of \\ Colorado Hospital, Aurora, CO, USA
}

Correspondence: Paul F Cook University of Colorado Denver, College of Nursing, Campus Box C288-04,

13120 E. 19th Avenue, Aurora, CO 80045 , USA

Tel +l 3037248537

Fax +I 3037248560

Email paul.cook@ucdenver.edu
Introduction: Adherence to glaucoma treatment is poor, potentially reducing therapeutic effects. A glaucoma educator was trained to use motivational interviewing (MI), a patientcentered counseling style, to improve adherence. This study was designed to evaluate whether MI was feasible in a busy ophthalmology practice.

Methods: Feasibility was assessed using five criteria from the National Institutes of Health Behavior Change consortium: fidelity of intervention components to MI theory; success of the training process; delivery of MI-consistent interventions by the glaucoma educator; patient receipt of the intervention based on enrollment, attrition, and satisfaction; and patient enactment of changes in motivation and adherence over the course of the intervention.

Results: A treatment manual was designed by a multidisciplinary team with expertise in health psychology, public health, and ophthalmology. The glaucoma educator received 6 hours of training including role-play exercises, self-study, and individual supervision. His MI-related knowledge and skills increased following training, and he delivered exclusively MI-consistent interventions in $66 \%$ of patient encounters. $86 \%(12 / 14)$ of eligible patients agreed to be randomized into glaucoma educator support or a control condition. All 8 patients assigned to the glaucoma educator completed at least 2 of 6 planned contacts, and $50 \%(4 / 8)$ completed all 6 contacts. Patients assigned to the glaucoma educator improved over time in both motivation and adherence.

Conclusion: The introduction of a glaucoma educator was feasible in a busy ophthalmology practice. Patients improved their adherence while participating in the glaucoma educator program, although this study was not designed to show a causal effect. The use of a glaucoma educator to improve glaucoma patients' medication adherence may be feasible at other ophthalmology clinics, and can be implemented with a standardized training approach. Pilot data show the intervention can be implemented with fidelity, is acceptable to patients and providers, and has the potential to improve adherence.

Keywords: adherence, counseling, glaucoma, medication, training

\section{Introduction}

Glaucoma is a leading cause of blindness worldwide. ${ }^{1,2}$ In the United States alone, over 2 million people have glaucoma, and 130,000 are legally blind from this disease. . $^{3,4}$ Unfortunately, medication nonadherence is an ongoing barrier to treatment. In one early study, glaucoma patients were just $42 \%$ adherent after being told they could go blind, and adherence improved only to $58 \%$ among patients who had already gone blind in one eye..$^{5}$ Although medications have improved since that time with fewer doses per day and decreased systemic side effects, nonadherence to current glaucoma medications is still close to $50 \%{ }^{6}$ 
Barriers to glaucoma treatment adherence include medication regimen characteristics, logistical issues like scheduling challenges, individual patient factors like memory problems, and poor doctor-patient communication. In a recent survey, $50 \%$ of ophthalmologists said that patients' lack of motivation for treatment was a barrier to adherence, 55\% said that medication cost reduced patients' adherence, and $41 \%$ said that patients' lack of knowledge about treatment was a primary barrier to adherence. ${ }^{7}$ However, studies of patients' actual adherence behavior have found only a modest association between treatment-related knowledge and adherence. ${ }^{8}$ Patients' negative attitudes toward treatment appear to be a more important determinant of nonadherence than lack of knowledge: patients who do not believe that nonadherence might lead to reduced vision are less likely to adhere to treatment, as are patients who report concerns about cost or trouble staying adherent while traveling. ${ }^{9}$ About one-third of glaucoma nonadherence is intentional, with patients being less likely to take medication if they see no need for it or are not concerned about the potential consequences of nonadherence. ${ }^{10}$ Demographic and clinical variables including gender, marital status, geographic area, and treatment duration do not predict adherence. ${ }^{8}$ However, age, minority race/ ethnicity, and comorbid medical illness have been found to predict nonadherence in some studies. ${ }^{11}$

\section{Motivational interviewing to improve medication adherence}

Support and education from medical professionals can increase adherence. ${ }^{12}$ One model of provider-delivered support is motivational interviewing (MI), a counseling style focused on exploration and resolution of patients' ambivalence. ${ }^{13}$ Several meta-analytic reviews ${ }^{14,15}$ demonstrate MI's utility for health behavior problems including nonadherence: It has been successfully used by primary care practitioners to promote healthy behaviors, ${ }^{16}$ by diabetes specialists to increase physical activity, ${ }^{17}$ and by dentists to improve oral health behaviors. ${ }^{18}$ In addition, nurse-delivered telephonic counseling using MI has been found to improve medication adherence for endometriosis, ${ }^{19}$ osteoporosis, ${ }^{20}$ serious and persistent mental illness, ${ }^{21} \mathrm{HIV}^{22}$ and ulcerative colitis. ${ }^{23}$ MI may be particularly helpful to patients from minority cultural groups. ${ }^{24}$

To our knowledge, MI has not been used in ophthalmology practice, a setting that presents several challenges. First, glaucoma care traditionally has been conducted directly between physicians and patients, with little involvement of ancillary staff like nurses or care managers. However, patients and their ophthalmology providers may have different perceptions of adherence. ${ }^{18}$ Second, counseling techniques like MI are less familiar to ophthalmology practitioners than to those in other specialties, and require a communication style that is substantially different from ophthalmologists' customary physician-directed approach. ${ }^{25}$ Third, patients may not be accustomed to receiving education or counseling from their ophthalmology providers. Finally, as in most care settings, lack of time and funding are barriers to change. ${ }^{26}$

\section{Can MI be delivered by a glaucoma educator in ophthalmology practice?}

This pilot study was designed to evaluate the feasibility of training a glaucoma educator to implement MI with patients at an outpatient ophthalmology practice. The rationale for adding a glaucoma educator was three-fold: (a) patients may benefit from extra time with a health care professional; (b) patients may feel more comfortable asking questions of an ancillary provider; ${ }^{27}$ and (c) educators can interact with patients using a style different from the traditional medical model. ${ }^{13}$ If MI delivered by a glaucoma educator improves adherence, this may also lead to reduced intraocular pressure, preservation of visual field, improved quality of life, delayed need for specialized or long-term care, and reduced overall health care costs. But because glaucoma educators and MI counseling are not currently part of ophthalmology practices, the feasibility of this approach must first be established.

Feasibility was evaluated using criteria from the NIH Behavior Change Consortium. ${ }^{28,29}$ This 5-level conceptualization suggests that an intervention is translated to a new setting with high fidelity if it has adequate adherence to theory, if it is implemented successfully, delivered consistently by interventionists, and successfully received by patients, and if patients enact recommended changes in behavior.

\section{Methods \\ Participants}

Participants were recruited from the tertiary glaucoma clinic of one of the authors (MYK) at the Rocky Mountain Lions Eye Institute, Denver, CO in 2008. Physicians identified adult patients with primary or secondary open-angle glaucoma who were prescribed monotherapy topical glaucoma medication. Exclusion criteria were: patient-reported inability to administer eye drops, cognitive impairment, physician's determination that glaucoma surgery was likely within 6 months, or $>80 \%$ adherence during a 2-month run-in phase. Eligible patients were randomized to receive either 
standard glaucoma care or standard care plus glaucoma educator counseling. Patients did not receive any incentives for participating in the study.

Fifty-one patients consented, and 14 were $<80 \%$ adherent. The high rate of adherence in $73 \%$ (37/51) of screened patients may have resulted from adherence monitoring during the run-in phase. Twelve of these patients consented to be randomized, and 8 were assigned to the glaucoma educator intervention. Patient recruitment and study flow are shown in Figure 1.

\section{Procedure}

This study was approved by the Colorado Multiple Institutional Review Board, and participants gave written informed consent. On average, 35-40 patients were seen per half day of clinic during the recruitment period. Patients were approached to participate by their ophthalmologist and those who agreed were escorted by the clinic study coordinator to a research examination lane where the consent process was completed. During the run-in phase and intervention, participants stored their medication eye-dropper in a bottle with a Medication Event Monitoring System (MEMS) cap. MEMS caps are electronic devices that record the time and date a pill bottle is opened. Bottles with MEMS caps accommodate all currently used glaucoma eye drops. Patients were given the MEMS bottle by the clinic study coordinator at the time they provided informed consent, and they returned to the clinic for a second meeting with the study coordinator at the end of the 2-month run-in period to determine baseline adherence

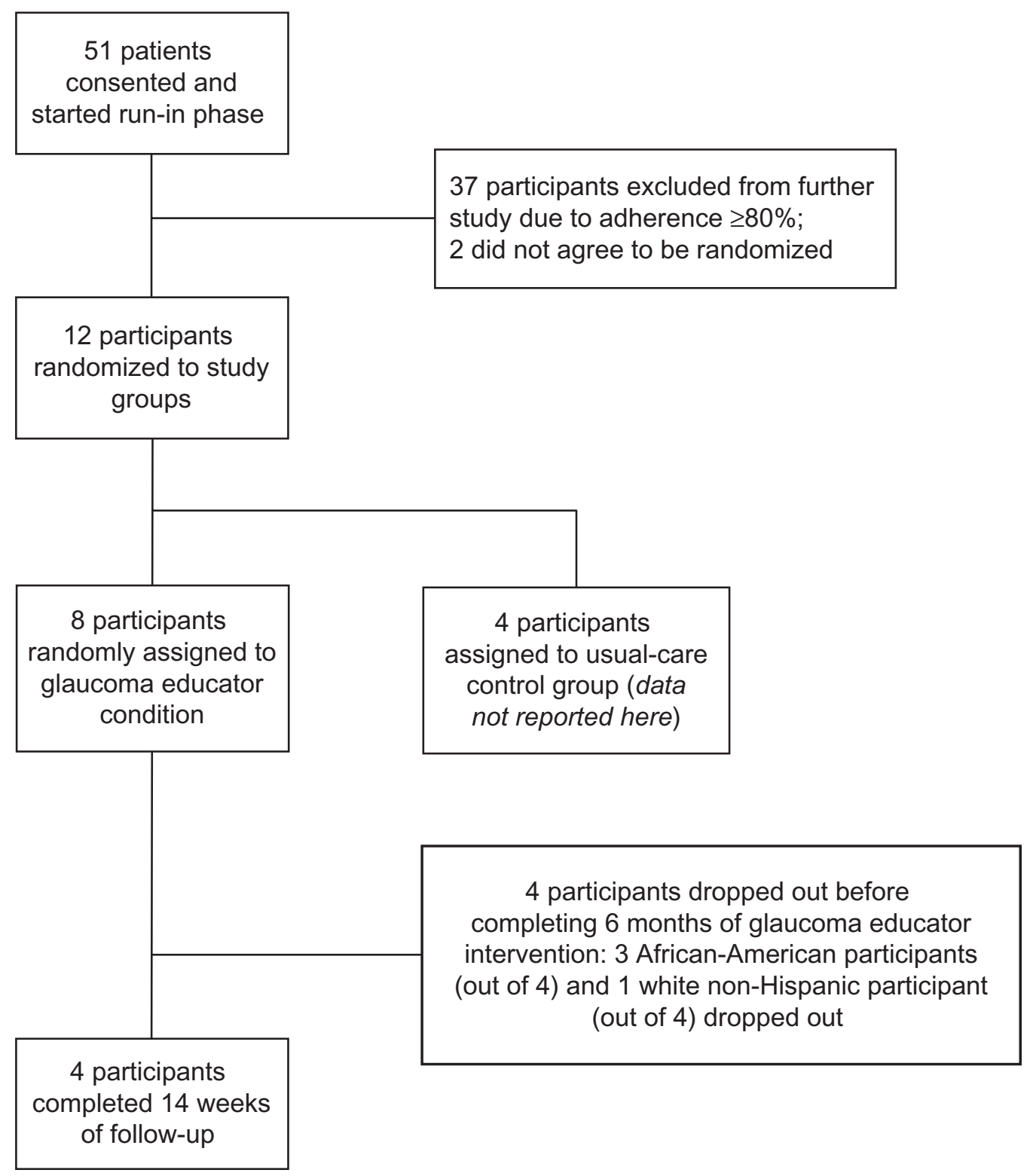

Figure I Study recruitment and participant flow. 
and study eligibility. Eligible patients randomized to the glaucoma educator condition were then scheduled for their initial educator visit at a later date. The glaucoma educator had no previous contact with any of the study participants. MEMS data were also downloaded and analyzed at each in-person glaucoma educator visit. Participants were aware that MEMS were being used to monitor their medication use. The long run-in period was designed to ensure that any improvements in adherence due to MEMS use occurred prior to the glaucoma educator intervention so that any further improvements in adherence would be attributable to the MI intervention.

Participants assigned to the glaucoma educator were scheduled to receive three one-to-one meetings with the glaucoma educator and three phone calls. Each included a review of the participant's current adherence, barriers to taking medication, side effects, and questions about treatment. The glaucoma educator was trained to recognize and address habits, beliefs, and emotions that interfere with adherence, and to respond within an MI counseling framework. ${ }^{19}$ To reinforce teaching points, the educator distributed print material approved by the American Academy of Ophthalmology.

The first glaucoma educator session was scheduled within 1 week after randomization. Follow-up calls were scheduled 1,6 , and 14 weeks later, with in-person meetings scheduled 4 and 12 weeks post-randomization. Patients continued to receive care from their ophthalmologists, who were masked to patients' group assignment.

\section{Measures}

\section{Theory fidelity}

MI is based on four principles, summarized in Table 1: express empathy, roll with resistance, develop discrepancy, and support self-efficacy. ${ }^{30}$ These principles define an empathic counseling style focused on listening carefully to individual patients' concerns, increasing patients' awareness about unintended consequences of behavior, avoiding argument or lecturing, and encouraging patients to develop their own solutions and make informed decisions about adherence. The empathic and directive "spirit" of MI is more important than specific counselor behaviors. ${ }^{31}$ A manual and counselor tools were developed for this intervention, and their fidelity to MI theory was evaluated by multidisciplinary expert review. The educator's feedback was also obtained.

\section{Glaucoma educator training}

The training process was documented, including the glaucoma educator's prior counseling experience, training received, and follow-up consultation. The educator's knowledge and comfort with the intervention were assessed using standardized educational evaluation tools. ${ }^{32}$

\section{Implementation of MI}

Implementation was documented using a session record form developed for this study. Session length and number of contacts were recorded. The glaucoma educator also reported his use of MI techniques, participants' current adherence, any barriers identified, and participants' readiness for change.

\section{Patient receipt of $\mathrm{MI}$}

Receipt is the degree to which patients receive the intervention as designed. Receipt was evaluated based on eligible patients' participation and attrition from the program over time.

\section{Patient enactment}

Enactment is the degree to which participants take necessary follow-up steps, such as increasing motivation or changing behavior. Motivation was assessed at each session via a stageof-change rating ${ }^{33}$ by the glaucoma educator, coded numerically on a 1-4 scale with 1 indicating the lowest readiness for change and 4 the highest. Adherence was based on MEMS data, which are widely regarded as valid, ${ }^{34}$ do not have a strong direct effect on patients' medication-taking behavior, ${ }^{35}$ and have been used in glaucoma research. ${ }^{7,28,29}$ Although MEMS data are considered as close to a "gold standard" as exists in the science of medication adherence, using multiple

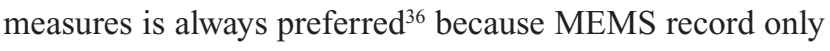
the first step in using medication (opening the bottle) rather than an actual attempt to administer the medication or verification that eye drops were administered correctly. Therefore, MEMS data were supplemented with a clinical interview measure that has shown $>70 \%$ agreement with pharmacy data. ${ }^{20}$ For both measures, adherence was defined as the percentage of days since the last session on which medication was taken as prescribed, based on the number of doses taken but not on the specific timing of doses. This adherence metric was selected to keep the two measures comparable, and to minimize recall bias in patient reports.

\section{Data analysis}

Analyses were primarily descriptive statistics, calculated using SPSS software (v. 17; SPSS, Inc., Chicago, IL, USA). For patient enactment measures, the multilevel modeling program HLM 6.03 (Scientific Software International Inc., Lincolnwood, IL) was used to evaluate within-patient changes in motivation and behavior over time. 
Table I Motivational interviewing (MI) counseling style

\begin{tabular}{|c|c|}
\hline MI principle & Sample communication techniques \\
\hline \multirow[t]{3}{*}{ Express empathy } & — Use open-ended rather than yes/no questions (“how are you doing with this medication?") \\
\hline & — Reflect back the patient's statements (“I hear you saying ...”) \\
\hline & $\begin{array}{l}\text { — Validate the patient's concerns ("a lot of people have trouble taking their medication exactly as } \\
\text { it's prescribed ...") }\end{array}$ \\
\hline \multirow[t]{4}{*}{ Roll with resistance } & — Don't argue (“I apologize; I wasn't trying to lecture you”) \\
\hline & — Reflect back emotions (“it sounds like you feel stuck”) \\
\hline & — Ask about the patient's experience ("what have you heard about this medication?" "are there \\
\hline & people in your family who had similar experiences?" "what have you tried so far?”) \\
\hline \multirow[t]{5}{*}{ Develop discrepancy } & — Empathize with ambivalence (“you want to, and it's also hard”) \\
\hline & — Highlight areas of contradiction ("there are things you don't like about treatment, but you also \\
\hline & see some benefits”) \\
\hline & — Summarize problems and strengths together ("you've had trouble remembering medication, but \\
\hline & you are able to juggle many other things in your life successfully") \\
\hline \multirow[t]{6}{*}{ Support self-efficacy } & — Emphasize autonomy ("this is really your decision”) \\
\hline & — Educate using "ask-tell-ask" rather than "tell-ask-tell” ("how do you usually take your medication? \\
\hline & Can I tell you a little more about the way it works? The medication only stays active for a certain \\
\hline & length of time, so it's very important to use the drops exactly 12 hours apart to get the desired \\
\hline & benefits. What do you think of what I just told you?") \\
\hline & - Ask the patient to make choices ("what would you like to do now? Where do we go from here?") \\
\hline
\end{tabular}

\section{Results}

Results for the five dimensions of treatment fidelity are summarized in Table 2.

\section{Theory fidelity}

The authors created an intervention manual with information about why traditional patient education is ineffective, basic MI strategies (eg, open-ended questions, acceptance, reflection, and summarizing), the "ask-tell-ask" method of patient education, additional techniques for patients who appear "resistant" to change, and problem-solving strategies for more motivated patients. A form was also developed to document counseling sessions.

The manual and session record form were reviewed by experts in health psychology, public health, and ophthalmology. Additional feedback was obtained from three colleagues with expertise in MI, each of whom has a terminal degree in nursing or social work and at least 5 years of clinical experience in health behavior change. All components were viewed as being consistent with the theory of behavior change underlying MI. ${ }^{24}$ Suggestions incorporated from this review focused on addressing logistical barriers to adherence and changes in the exact wording of interventions. Additional feedback was requested from the glaucoma educator, who requested further information about cultural competence and mental health issues that might interfere with adherence. These topics were incorporated into an additional training session and practice exercises.

\section{Glaucoma educator training}

The glaucoma educator was a certified ophthalmic technician with over 12 years of clinical experience. He completed training with two ophthalmologists on glaucoma drops and their side effects. He then reviewed the intervention manual and completed in-person training with one of the authors (PFC), a psychologist with expertise in health behavior change. Training included discussion of the manual, role-playing patient scenarios, and individual consultation to discuss the first few patients seen. Training that includes face-to-face instruction plus supervised practice over time is more efficacious than single-day workshops or self-study alone. ${ }^{37}$

At the start of training, the educator had excellent interpersonal skills and some patient education experience, but no experience using MI. By the end of training, he almost exclusively used MI-consistent techniques during role-play and always elicited the patient's knowledge and reactions before offering educational messages. The trainer's ratings of the educator's MI skills reflected this improvement. In addition, the educator's self-assessed knowledge of MI, willingness to use MI, and reported use of eight MI-consistent techniques each increased from pre- to post-training.

\section{Implementation of MI}

Based on the session record form, the counselor used MI-consistent listening techniques $100 \%$ of the time. For patients with low readiness for change, all 13 counseling sessions involved elicitation, reflection, and summarization of participant responses, and 10 also included patient 
Table 2 Treatment fidelity of motivational interviewing (MI) in outpatient glaucoma care

\begin{tabular}{|c|c|c|}
\hline Criteria & Fidelity measures & Results \\
\hline Theory fidelity & $\begin{array}{l}\text { I. Expert review by co-authors and three colleagues } \\
\text { with MI expertise } \\
\text { 2. Feedback from glaucoma educator }\end{array}$ & $\begin{array}{l}\text { I \& } 2 \text {. Suggestions led to new material on logistical } \\
\text { barriers to adherence, cultural competence, and mental } \\
\text { health issues, plus some changes in the exact wording of } \\
\text { interventions }\end{array}$ \\
\hline Educator training & $\begin{array}{l}\text { I. Amount of training provided } \\
\text { 2. Trainer rating of educator's } \mathrm{MI} \text { skill on a I-IO scale } \\
\text { (I = no experience; } 4=\text { novice; } 7=\text { skilled; } \\
\text { and IO= expert) } \\
\text { 3. Educator's ratings on Likert-type scales } \\
\text { ( } \mathrm{I}=\text { low to } 5=\text { high): knowledge, } \mathrm{MI} \text { skills, } \\
\text { and willingness to use } \mathrm{MI}\end{array}$ & $\begin{array}{l}\text { I. Training included } 5.5 \text { hours self-study }+6 \text { hours in-person } \\
\text { with role-plays } \\
\text { 2. Trainer's ratings: educator's } \mathrm{Ml} \text { skill at start of } \\
\text { training }=3, \mathrm{MI} \text { skill after training }=7 \\
\text { 3. Educator's ratings: increase in knowledge (pre }=1.5 \text {, } \\
\text { post }=4.5 \text { ), MI skills (pre }=I .3 \text {, post }=2.5 \text { ), and } \\
\text { willingness (pre }=I .3 \text {, post }=4.0 \text { ) }\end{array}$ \\
\hline Implementation & $\begin{array}{l}\text { I. Counselor's use of Ml-consistent interventions, } \\
\text { as documented on session record form. } \\
\text { Interventions were considered MI-consistent } \\
\text { if they matched the patient's counselor-rated } \\
\text { stage of readiness for change }\end{array}$ & $\begin{array}{l}\text { I. For patients with low readiness: I3/I3 sessions used } \\
\text { active listening alone (MI-consistent), I0/I } 3 \text { also used } \\
\text { problem-solving (MI-inconsistent). For patients with } \\
\text { high readiness: I I/I6 sessions used active listening, 2/I6 } \\
\text { education plus listening, and 3/I6 problem-solving plus } \\
\text { listening (all Ml-consistent) }\end{array}$ \\
\hline Receipt & $\begin{array}{l}\text { I. Enrollment } \\
\text { 2. Attrition } \\
\text { 3. Representativeness of participating } \\
\text { patients }\end{array}$ & $\begin{array}{l}\text { I. I } 2 / 14 \text { eligible patients consented } \\
\text { 2. } 8 / 8 \text { patients completed } 2+\text { sessions; } 4 / 8 \text { patients } \\
\text { completed all } 6 \text { sessions } \\
\text { 3. } 4 \text { participants }(50 \%) \text { were male, and } 4(50 \%) \text { were white } \\
\text { non-Hispanic; clinic patients overall were } 38 \% \text { male and } \\
78 \% \text { white non-Hispanic }\end{array}$ \\
\hline Enactment & $\begin{array}{l}\text { I. Change in motivation, based on a I-4 } \\
\text { stage-of-change scale } \\
\text { 2. Change in \% adherence, based on MEMS } \\
\text { and clinical interview measures }\end{array}$ & $\begin{array}{l}\text { I. Motivation increased with more sessions, } T=1.98 \text {, } \\
P=0.058, \beta=0.15 \\
\text { 2. Adherence increased based on MEMS, } T=2.25, P=0.032 \text {, } \\
\beta=2.68 \text {, but not based on clinical interview, } T=1.07, \\
P=0.30, \beta=1.03 \text {. }\end{array}$ \\
\hline
\end{tabular}

education (considered MI-consistent as long as it was offered together with active listening techniques). However, 10 of these 13 sessions also involved active problem solving, which was considered an MI-inconsistent technique for this patient group. With patients who had higher readiness for change, 11 of 16 sessions involved listening alone, 2 involved listening with education, and 3 involved listening with both education and action-oriented strategies, all of which were considered MI-consistent. Overall, the educator used MI-consistent techniques in all sessions, and used exclusively MI-consistent techniques in 19/29 sessions $(66 \%)$.

\section{Patient receipt of $\mathrm{MI}$}

Of 14 eligible patients, 12 (77\%) agreed to be randomized; the two patients who did not agree were both African-American women, one aged 57 years and one over 80 years. Patients in the glaucoma educator condition had an average age of 57.9 years (standard deviation $[\mathrm{SD}]=8.5)$, and $4(50 \%)$ were male. Participants were 50\% (4) white non-Hispanic and 50\% (4) African-American. The demographics of the four patients randomly assigned to the control condition were similarly diverse: these participants had an average age of 55.5 years $(\mathrm{SD}=14.7), 3(75 \%)$ were male, and only $1(25 \%)$ was white, with one Asian, one African-American, and one Pacific Islander participant in the control group. The demographics of patients randomly assigned to the glaucoma educator were more diverse than the total clinic population, where patients were 38\% male and $78 \%$ white non-Hispanic. It is not known why the sample included more men and African-American patients, as these groups were not specifically targeted for recruitment. However, this finding does suggest that the glaucoma educator intervention was acceptable to a diverse patient group. Participants were selected specifically for nonadherence, which may be correlated with nonwhite race/ethnicity. Baseline nonadherence was high both for patients randomly assigned to the glaucoma educator (MEMS-based nonadherence on $M=36.7 \%$ of days per week, $\mathrm{SD}=18.5$ ) and for those assigned to the control condition (nonadherence on $M=37.0 \%$ of days per week, $\mathrm{SD}=12.9$ ).

The acceptability of MI to patients is illustrated by the following case vignette:

$\mathrm{Mr} \mathrm{S}$ was a 52-year-old African-American man. His baseline adherence was $61 \%$, and when offered a chance to speak 
with the glaucoma educator, he said he appreciated someone taking time to answer his questions. Mr S assumed he needed to take his medication "at bedtime," which varied widely from day to day. Mr S also said that he was often tired and forgot to use his drops at night. The glaucoma educator used the "ask-tell-ask" method to teach Mr S about the importance of dosing every 24 hours, and that the time of day was flexible. Mr S expressed frustration that no one had explained this to him before, and the glaucoma educator validated $\mathrm{Mr}$ S's desire to manage his own treatment more effectively. $\mathrm{Mr} \mathrm{S}$ offered his own solution of taking medication at $8 \mathrm{pm}$ as opposed to "at bedtime." Further contacts helped Mr S to maintain this static time strategy and to overcome difficulties with forgetting. Based on these changes, $\mathrm{Mr} \mathrm{S}$ improved his adherence to $95 \%$ by the end of 3 months of contact with the glaucoma educator. $\mathrm{Mr} \mathrm{S}$ said he was very satisfied with the new therapeutic dosing regimen and with the assistance he received from the educator.

Of the eight patients randomly assigned to the glaucoma educator, all eight completed at least two sessions. On average, participants received $4.5(\mathrm{SD}=1.85,95 \%$ confidence interval [CI]: $2.95,6.05)$ of six planned contacts, including $2.25(\mathrm{SD}=0.89)$ in-person and $2.0(\mathrm{SD}=0.93)$ telephone contacts. In-person sessions lasted 30-45 minutes, and telephone sessions lasted 5-10 minutes. Attrition was 4/8 (50\%) over 6 months. Older patients were more likely to attrit, $r=0.52$, and more African-American (75\%) than white participants $(25 \%)$ did not complete the full 6-month intervention. Two of the four participants who dropped out stated that they were unwilling to make further study-related clinic visits in the absence of incentives, and the other two participants were lost to follow-up due to a lack of stable contact information - both of these patients had disconnected phones and also failed to return for usual care. In one case, the participant was sent a registered letter, which was also not received. However, no participants reported any adverse events, and no patients withdrew specifically because they were dissatisfied with the intervention.

\section{Patient enactment}

Patient behavior was analyzed using an intent-to-treat method, with all patients in the glaucoma educator condition included in analyses regardless of how many sessions they completed. Participants' readiness for change and two measures of adherence were modeled as separate outcomes, each analyzed within persons based on the number of glaucoma educator sessions completed. Models corrected for moderate to high inter-correlation of data points within participants, intraclass correlation $(I C C)=0.49$ for readiness, $I C C=0.75$ for counselor-rated adherence, and $I C C=0.77$ for MEMSbased adherence. Effects are reported as unstandardized betas on a $1-4$ scale for readiness, and as 0-100 percentages for the two adherence measures. As shown in Table 1, participants' readiness for change increased as more educator visits were completed. Participants' adherence improved significantly over time based on MEMS data, with no significant change in counselor-rated adherence. MEMS are likely the more accurate measure, as counselor-rated adherence was initially higher and may have been biased by a ceiling effect due to inaccurate patient reporting of adherence.

Patient enactment of behavior change is illustrated by the following case vignette:

Ms D was a 60-year-old white female. Her baseline adherence was $75 \%$, which she was surprised to learn during her first visit with the glaucoma educator. When asked how she felt about her current adherence, Ms D said she was disappointed, and that difficulty using her eye drops and forgetfulness were the primary reasons for her poor adherence. The glaucoma educator responded by asking about Ms D's goals and past experiences. Ms D noted that she did take other medications successfully, even when traveling. She set a goal of $>85 \%$ adherence for herself, and suggested that using eye drops at the same time as her other evening medications might help her to remember them. The glaucoma educator agreed to a test of this plan. Over 3 months of follow-up contact, the glaucoma educator also helped Ms D problem-solve difficulties in administering eye drops by finding a technique that worked for her. Ms D was able to improve her adherence to $100 \%$ by the end of the program. Over time, it also emerged that Ms D had not strongly believed treatment was beneficial; the glaucoma educator helped her to explore this ambivalence, and by the conclusion of the program Ms D said that conversations with the educator had helped her to see the importance of taking medication correctly.

\section{Discussion}

Medication nonadherence is a barrier to successful glaucoma treatment that is not systematically addressed in most ophthalmology practices. The current study demonstrated that an in-person and telephone glaucoma educator intervention was feasible for implementing MI, a research-based health behavior change counseling technique, in a busy ophthalmology practice. Using an intervention manual and clinical support tools developed by a multidisciplinary expert group, a glaucoma educator was trained to implement MI with nonadherent patients. The educator had no initial exposure 
to $\mathrm{MI}$, but reported that the method was relatively easy to learn. After individual training and self-study, his self- and instructor-rated competence with these methods improved from novice to skilled. Session rating forms showed that all of his interventions used MI-consistent techniques, but that he over-used problem-solving techniques with participants who were not yet ready for change. This is a common issue among providers new to MI, and additional training on matching techniques to patients' readiness for change might improve these results. However, research also shows that multiple counselor behaviors may be appropriate, as long as they are delivered in the patient-centered "spirit" of MI. ${ }^{31}$

Ophthalmology patients were willing to participate in the educator intervention. Participants' demographics were more diverse than the overall population served by this clinic, indicating that the intervention was acceptable to a broad range of glaucoma patients. Half of patients dropped out early, so attrition was a significant limitation to MI's feasibility in this study. The requirement of $<80 \%$ baseline adherence may have resulted in a particularly nonadherent sample who were more difficult to retain over time. Two of the four participants lost to follow-up had their phone numbers disconnected and left care completely at the time they dropped out of the study. Although 50\% attrition over six planned contacts is not unusual for psychosocial interventions, ${ }^{38}$ it was considered quite high for this ophthalmology practice. Despite the high attrition rate, all participants received at least two of six planned contacts, which was considered an adequate dose of the intervention because even brief MI is efficacious. ${ }^{14}$

Given that the intervention was successfully implemented and acceptable to patients, a final question is whether participants were able to enact recommended changes. Participants showed nonsignificant improvement in readiness for change and significant improvement in MEMS-based adherence over the course of the intervention, although there was no change on counselor-rated adherence. Whether these changes represented improvements over standard care is an efficacy question not addressed in the current study.

Potential challenges to widespread adoption of the glaucoma educator intervention in ophthalmology practice include staffing the educator position and integrating behavior change techniques with other aspects of medical care. Although in this study ophthalmologists were masked to the educator intervention, in actual practice the educator would be in regular contact with the patient's ophthalmologist. This might enhance benefits of the intervention due to better coordination of care, or might reduce its effects if patients are less honest or less able to develop a working relationship with the glaucoma educator.

Cost of the educator position is another potential concern. However, if a glaucoma educator improves adherence and reduces long-term costs (eg, due to reduced vision loss, hospitalization, or need for long-term care), then a case could be made to insurers and other stakeholders that this service should be reimbursed. Health and behavior CPT codes exist to categorize patient counseling for chronic disease self-management, so an effective billing mechanism is not the issue, only whether adherence counseling should be a covered benefit. Cost-effectiveness analyses are therefore an important focus for future work.

\section{Limitations and directions for future research}

This study found that a glaucoma educator intervention was feasible in one outpatient ophthalmology practice. A small sample size and single clinic setting are important limitations to the generalizability of results. The two participating ophthalmologists were already aware of the problem of glaucoma medication nonadherence and committed to finding solutions. Implementation may be more difficult in settings where ophthalmologists are less aware of or interested in addressing nonadherence. Results showed that a newly trained glaucoma educator was relatively successful in delivering MI-consistent interventions although he also delivered some MI-inconsistent interventions. Conclusions about the actual content of glaucoma educator interventions would be strengthened in future studies by incorporating an independent expert's ratings of session tapes or transcripts using an objective behavioral coding system such as the Motivational Interviewing Skills Code, ${ }^{39}$ in addition to the glaucoma educator's report.

Measurement is a universally acknowledged limitation in adherence studies, and the current investigation was no exception. The use of multiple measures is recommended because there is no "gold standard" for adherence. ${ }^{36}$ Although we used two independent measures of adherence - MEMS and clinical interview - that have each been used in past research and are supported by psychometric data, the two measures did not always agree. In our study, adherence based on MEMS tended to be lower than educator-rated adherence. This may in part explain the finding that adherence improved based on MEMS data only, because MEMSbased adherence had more room to improve. This finding also highlights health care providers' challenges in making accurate judgments about adherence: Recent research suggests 
that ophthalmologists detect nonadherence in less than 30\% of cases where the patient is actually nonadherent. ${ }^{25}$ Both adherence measures in this study assessed only the percentage of days on which patients took the correct number of doses; because glaucoma medications have short half-lives and the timing of doses is also important, more fine-grained MEMS-based adherence measures of dose timing may be desirable in future research.

Although participants were aware that MEMS were being used to monitor their adherence, research in other fields suggests that participants are more likely to misrepresent their adherence verbally than to deliberately falsify data by opening MEMS devices without taking medication. ${ }^{35}$ MEMS may produce some improvement in adherence and of themselves, but this study's 2-month run-in period helped to ensure that any patients who improved their adherence due to mere measurement effects were excluded prior to the start of the intervention. Nevertheless, unobtrusive adherence measures such as pharmacy fill data would strengthen further research by eliminating concerns about measurement effects.

Attrition was an important limitation to conclusions about patients' receipt and enactment of MI. It is important to note that African-American patients were more likely to drop out, and also have been found to have lower adherence in previous research. ${ }^{11}$ Based on analyses that included all available data from both patients who completed the full intervention and those who dropped out, patients increased their motivation and adherence while participating in the educator program, but this finding requires replication.

Finally, this feasibility study was not designed to prove that improvements in adherence were causally related to the educator program. Attrition, history effects, maturation, regression to the mean, or other artifacts are potential competing explanations. The current study also was not designed to differentiate the specific effects of MI from those that might be achieved by mere attention from a glaucoma educator. These limitations will be most effectively addressed in future work comparing participants' results to a randomized control group.

\section{Implications for practice}

Glaucoma medications improve long-term outcomes, but patients find adherence difficult, and nonadherence increases the chance of disease progression. Following the lead of other medical specialties, the authors adopted a team approach to improve adherence. A glaucoma educator supported patients over time using MI. Working with local health behavior experts facilitated the integration of MI into ophthalmology practice. Although experts in using MI to promote adherence may not be available in all clinical settings, many community-based mental health practitioners have expertise in this approach and an interest in integrating psychological counseling into medical settings. ${ }^{41}$ To identify mental health professionals with relevant expertise, US ophthalmologists can consult online listings at http://www. findapsychologist.org/ or http://www.therapytribe.com/, or find their local community mental health center at http:// mentalhealth.samhsa.gov/databases/.

In this study, MI techniques were successfully learned and delivered by a glaucoma educator with no prior training in patient counseling methods. Not all practices may be able to afford or implement a separate glaucoma educator position. Training ophthalmologists themselves to use MI might be another option, although one that was not evaluated in this study. Some evidence does suggest that ophthalmologists can learn a more patient-centered counseling style and that it improves their ability to detect nonadherence. ${ }^{40}$ However, because physician time is more expensive than nonphysician time and not all ophthalmologists may want to change their counseling style, ${ }^{7,25}$ feasibility may be greater when MI is provided by a separate educator. Patients also may be more honest with nonphysician health care providers. $^{42}$

The current study delivered MI only to a subgroup of patients selected for poor adherence based on MEMS data during a 2-month run-in period. This procedure limits generalizability of the findings to nonadherent glaucoma patients only. In general practice, it would be preferable to offer MI proactively to all patients prescribed glaucoma medication rather than offering support only after nonadherence occurs. This is especially true because almost $50 \%$ of patients with glaucoma are nonadherent, ${ }^{6}$ and ophthalmologists have difficulty detecting nonadherence. ${ }^{25}$

Participants received a moderate dose of MI over 6 months, although there were some problems with the match between counseling strategies and participants' readiness for change. Attrition was also a potential problem, with a sample that was selected specifically for nonadherence and a higher percentage of African-American than white participants leaving the educator intervention early. Nevertheless, all participants received an adequate dose of MI based on prior meta-analytic findings. ${ }^{14}$ Furthermore, MI was associated with increased readiness for change and improved adherence, and patient counseling in general has a significant dose-response effect, ${ }^{43}$ so multiple patient contacts are still 
recommended for MI interventions despite this study's high attrition rate. Overall, MI counseling delivered by a glaucoma educator appears to be a feasible adherence intervention in ophthalmology practice.

\section{Acknowledgments}

This study was sponsored by the American Glaucoma Society through an unrestricted educational grant from Pfizer. The authors wish to thank Courtney Lupia-Blasi for assistance with data entry and validation.

\section{Disclosure}

The authors report no conflicts of interest in this work.

\section{References}

1. Hyman L, Wu SY, Connell AM, et al. Prevalence and causes of visual impairment in the Barbados Eye Study. Ophthalmology. 2001;108(10):1751-1756.

2. Quigley HA. Number of people with glaucoma worldwide. $\mathrm{Br} J \mathrm{Oph}-$ thalmol. 1996;80(5):389-393.

3. Friedman DS, Wolfs RC, Colmain BJ, et al. Prevalence of openangle glaucoma among adults in the United States. Arch Ophthalmol. 2004;122(4):532-538.

4. Quigley HA, Vitale S. Models of open-angle glaucoma prevalence and incidence in the United States. Invest Ophthalmol Vis Sci. 1997;38(1):83-91.

5. Meichenbaum DC, Turk D. Facilitating Treatment Adherence: A Practitioner's Guidebook. New York, NY: Plenum Press; 1987.

6. Okeke CO, Quigley HA, Jampel HD, et al. Adherence with topical glaucoma medication monitored electronically: the Travatan Dosing Aid Study. Ophthalmology. 2009;116(2):191-199.

7. Gelb L, Friedman DS, Quigley HA, et al. Physician beliefs and behaviors related to glaucoma treatment adherence: the glaucoma adherence and persistency study. J Glaucoma. 2008;17(8):690-698.

8. Khandekar R, Shama ME, Mohammed AJ. Noncompliance with medical treatment among glaucoma patients in Oman - a cross-sectional descriptive study. Ophthalmic Epidemiol. 2005;12(5):303-309.

9. Friedman DS, Hahn SR, Gelb L, et al. Doctor-patient communication, health-related beliefs, and adherence in glaucoma: results from the glaucoma adherence and persistency study. Ophthalmology. 2008;115(8):1320-1327.

10. Rees G, Leong O, Crowston JG, Lamoureux EL. Intentional and unintentional nonadherence to ocular hypotensive treatment in patients with glaucoma. Ophthalmology. 2010;117(5):903-908.

11. Friedman DS, Okeke CO, Jampel HD, et al. Risk factors for poor adherence to eyedrops in electronically monitored patients with glaucoma. Ophthalmology. 2009;116(6):1097-1105.

12. Haynes RB, Yao X, Degani A, Kripalani S, Garg A, McDonald HP. Interventions for enhancing medication adherence. Cochrane Database Syst Rev. 2005 Oct 19;(4):CD000011.

13. Rollnick S, Miller WR, Butler CC. Motivational Interviewing in Health Care: Helping Patients Change Behavior. New York, NY: Guilford; 2007.

14. Burke BL, Arkowitz H, Menchola M. The efficacy of motivational interviewing: a meta-analysis of controlled clinical trials. $J$ Consult Clin Psychol. 2003;71(5):843-861.

15. Rubak S, Sandboek A, Lauritzen T, Christensen B. Motivational interviewing: a systematic review and meta-analysis. Br J Gen Pract. 2005;55(4):305-312.
16. Hecht J, Borrelli B, Breger RKR, DeFrancesco C, Ernst D, Resnicow K. Motivational interviewing in community-based research: experiences from the field. Ann Behav Med. 2005;29 Suppl:29-34.

17. Batik O, Phelan EA, Walwick JA, Wang G, LoGerfo JP. Translating a community-based motivational support program to increase physical activity among older adults with diabetes at community clinics: a pilot study of physical activity for a lifetime of success (PALS). Prev Chron Dis [serial on the Internet]. 2008;5(1):[7 p.]. http://www.cdc.gov/pcd/ issues/2008/jan/pdf/07_0142.pdf. Accessed Jun 10, 2010.

18. Weinstein P, Harrison R, Benton T. Motivating mothers to prevent caries: confirming the beneficial effect of counseling. J Am Dental Assoc. 2006;137(6):789-793.

19. Cook PF. Adherence to medications. In: O'Donohue WT, Levensky ER, editors. Promoting Treatment Adherence: A Practical Handbook for Health Care Providers. Thousand Oaks, CA: Sage; 2006. p. 183-202.

20. Cook PF, Emiliozzi S, McCabe M. Telephone counseling to improve osteoporosis treatment adherence: an effectiveness study in community practice settings. Am J Med Qual. 2007;22(6):445-456.

21. Cook PF, Emiliozzi S, Waters C, El-Hajj D. Effects of telephone counseling on antipsychotic adherence and ED utilization. Am J Manag Care. 2008;14(12):841-846.

22. Cook PF, McCabe MM, Emiliozzi S, Pointer L. Telephone nursing intervention improves adherence to medication for HIV. J Assoc Nurses AIDS Care. 2009;20(4):316-325.

23. Cook PF, Emiliozzi S, El-Hajj D, McCabe MM. Telephone nurse counseling for medication adherence in ulcerative colitis: a preliminary study. Patient Educ Couns. Epub 2010 Jan 13.

24. Miller WR, Rose GS. Toward a theory of motivational interviewing. Am Psychol. 2009;64(6):527-537.

25. Friedman DS, Hahn SR, Quigley HA, et al. Doctor-patient communication in glaucoma care: Analysis of videotaped encounters in community-based office practice. Ophthalmology. 2009;116(12): 2277-2285.

26. Prochaska JO, Prochaska JM. Why don't continents move? Why don't people change? J Psychother Integr. 1999;9(1):83-102.

27. Book HE. Some psychodynamics of non-compliance. Can J Psychiatry. 1987;32(2):115-117.

28. Resnick B, Bellg AJ, Borrelli B, et al. Examples of implementation and evaluation of treatment fidelity in the BCC studies: where we are and where we need to go. Ann Behav Med. 2005;29 Suppl:46-54.

29. Bellg AJ, Borrelli B, Resnick B, et al. Enhancing treatment fidelity in health behavior change studies: Best practices and recommendations from the NIH Behavior Change Consortium. Health Psychol. 2004;23(5):443-451.

30. Miller WR, Rollnick S. Motivational Interviewing. 2nd ed. New York, NY: Guilford; 2002.

31. Moyers TB, Miller WR, Hendrickson SML. How does motivational interviewing work? Therapist interpersonal skill predicts client involvement within motivational interviewing sessions. J Consult Clin Psychol. 2005;73(4):590-598.

32. Cook PF, Friedman R, Lord A, Bradley-Springer LA. Outcomes of multi-modal training for healthcare professionals at an AIDS Education and Training Center. Eval Health Prof. 2009;32(1):3-22.

33. Prochaska JO, Norcross J, DiClemente C. Changing for Good. New York: Avon Books; 1995.

34. Schwartz GF. Compliance and persistency in glaucoma follow-up treatment. Curr Opin Ophthalmol. 2005;16(2):114-121.

35. Lu M, Safren SA, Skolnik PR, et al. Optimal recall period and response task for self-reported HIV medication adherence. AIDS Behav. 2008;12(1):86-94.

36. Chesney MA. The elusive gold standard: future perspectives for HIV adherence assessment and intervention. J Acquir Immune Defic Syndr. 2006;43 Suppl 1:S149-S155.

37. Miller WR, Yahne CE, Moyers TB, Martinez J, Pirritano M. A randomized trial of methods to help clinicians learn motivational interviewing. J Consult Clin Psychol. 2004;72(6):1050-1062. 
38. Hansen NB, Lambert MJ, Forman EM. The psychotherapy doseresponse effect and its implications for treatment delivery services. Clin Psychol Sci Pract. 2002;9(3):329-343.

39. Moyers T, Martin T, Catley D, Harris KJ, Ahluwalia JS. Assessing the integrity of motivational interviewing interventions: reliability of the Motivational Interviewing Skills Code. Behav Cogn Psychother. 2003;31(2):177-184.

40. Hahn SR, Friedman DS, Quigley HA, et al. Effect of patient-centered communication training on discussion and detection of nonadherence in glaucoma. Ophthalmology. 2010;117(7):1339-1347.
41. Clay R. A new vision for American health care. APA Monitor. 2009;40(4):16.

42. Valenti WM. Treatment adherence improves outcomes and manages costs. The AIDS Reader. 2001;11(2):77-80.

43. Lambert MJ, Archer A. Research findings on the effects of psychotherapy and their implications for practice. In: Goodheart CD, Kazdin AE, Sternberg RJ, editors. Evidence-Based Psychotherapy: Where Practice and Research Meet. Washington, DC: American Psychological Association; 2006. p. 111-130.
Clinical Ophthalmology

\section{Publish your work in this journal}

Clinical Ophthalmology is an international, peer-reviewed journal covering all subspecialties within ophthalmology. Key topics include: Optometry; Visual science; Pharmacology and drug therapy in eye diseases; Basic Sciences; Primary and Secondary eye care; Patien Safety and Quality of Care Improvements. This journal is indexed on

Submit your manuscript here: http://www.dovepress.com/clinical-ophthalmology-journal

\section{Dovepress}

PubMed Central and CAS, and is the official journal of The Society of Clinical Ophthalmology (SCO). The manuscript management system is completely online and includes a very quick and fair peer-review system, which is all easy to use. Visit http://www.dovepress.com/ testimonials.php to read real quotes from published authors. 